\title{
VISCOUS HEATING IN PLANAR COUETTE FLOW: SERIES SOLUTIONS FOR TEMPERATURE-SENSITIVE FLUIDS
}

\author{
K.A. CARIDIS, B. LOUWAGIE \\ AND T.D. PAPATHANASIOU \\ Department of Chemical Engineering \& Chemical Technology, \\ Imperial College of Science and Technology, London SW7, UK
}

Key Words: Viscous Heating, Temperature-Sensitive Fluids, Rheological Corrections, Couette, Shear Flow

\begin{abstract}
The problem of the interaction between viscous heating, heat transfer and flow in Couette rheometers is investigated. Series solutions, up to $4^{\text {th }}$ order in the Brinkman number $(\mathrm{Br})$, are developed for planar Couette flows of materials whose viscosity and thermal conductivity can be expressed as polynomial functions of temperature with arbitrary coefficients. The derived solutions are validated by extensive comparison to existing closed-form results and to numerical solutions and are found to be highly accurate for a practical range of $(\mathrm{Br})$. The presented solutions provide valuable insight into the functionality of the relevant dependencies, something that is lost in purely numerical solutions. Based on these results, the distribution of velocity, shear rate and temperature, as well as their sensitivity on material and process parameters are investigated. Finally, corrections for viscosity measurements in Couette viscometers, which account for viscous heating and temperature-dependent material properties, are derived.
\end{abstract}

\section{Introduction}

The interaction between viscous heating and flow is of importance in a number of applications involving flow of materials with temperaturedependent properties. These include polymer processing (Agassant et al., 1991; Jansen and van Dam, 1992), tribology and lubrication (Galvin et al., 1981), food processing (Holdsworth, 1993; Harper, 1981), on-line measurements and instrumentation (Shadid and Eckert, 1992) and viscometry (Bird et al., 1960; Turian and Bird, 1963; Laun, 1983; Ko and Lodge, 1991). In the latter area, viscous heating is always a possible, and frequently significant, source of error in viscometric measurements at high shear rates, particularly with rotational viscometers, where the entire sample is sheared continuously throughout the duration of the measurement.

Many attempts have been made to obtain analytical solutions for flow in rotational viscometers that account for viscous heating and temperature dependent material properties. A rather complete review of early work in the field has been given by Sukanek and Lawrence (1974). When the thermal conductivity of the fluid is constant and the viscosity is an exponential function of temperature, an analytical solution for planar Couette flow has been presented by Nahme (1940) and quoted by Turian and Bird (1963). Gavis and Lawrence (1968) re-visited the planar Couette flow and, after formulating the problem in terms of the wall shear stress, determined that two

\footnotetext{
* Received on July 29, 1996. Correspondence concerning this
} article should be addressed to T.D. Papathanasiou. solutions exist for each value of the shear stress but that the solution is unique in terms of the Brinkman number. Gavis and Lawrence (1968) proceeded to present solutions for both the planar and the circular Couette flows under the same conditions as those used by Nahme (1940).

All previous analytical solutions have considered the thermal conductivity of the fluid to be independent of temperature. However, there is a number of current applications in which is not necessarily a correct assumption. The effect of temperature on the thermal conductivity of refrigerant fluids, for example, is not negligible (Tsvetkov et al., 1994). Powder-like materials are much more sensitive: the thermal conductivity of wet soil was found to increase by 3 to 5 times when the temperature increased from ambient to $90^{\circ} \mathrm{C}$ (Campbell et al., 1994) and a similar if not so spectacular trend has been observed in food-related moist powders (Halliday et al., 1995). Furthermore, recent non-equilibrium molecular dynamics simulations for liquid butane under shear (Daivis and Evans, 1995) have predicted the development of a non-isotropic thermal conductivity whose $(x x)$ and $(y y)$ components increase with shear rate. No exact analytical solutions are available for the corresponding problem. Bird et al. (1960), Turian and Bird (1963) and Turian (1965, 1969) have presented a methodology (attributed to Professor L. J. F. Broer, 1958) for obtaining approximate analytical solutions, in the form of series expansions in the Brinkman number $(B r)$, to the problem of combined flow and heat transfer for materials whose viscosity and thermal conductivity are polynomial functions of temperature. They then 
proceeded to develop such solutions, up to secondorder in $(B r)$, in the case of planar Couette flow. This result was applied to develop corrections in the coneand-plate viscometer. These solutions are explicit, and, even though they appear complicated, they can be easily programmed without recourse to numerical analysis. The rheological corrections derived from these solutions have been tested against experimental data and the solutions themselves were found to coincide with the first two terms of a Taylor expansion of Nahme's solution (1940). However, the range of $\mathrm{Br}$ ) for which these, necessarily approximate, solutions are accurate was not tested in detail.

The present contribution applies the same method and develops solutions for planar Couette flow up to $4^{\text {th }}$ order in $(B r)$. These are shown to exhibit an extended range of validity as compared to the solutions of Turian and Bird (1963) and also allow for a higher-order representation of the temperature effect on fluid viscosity. It should be pointed out that existing closed-form solutions consider the fluid viscosity to be analytically an exponential function of temperature. Determination of the relevant constant ( $\lambda$ in Eq. (35)) involves fitting experimental $\mu$ vs. $\Theta$ data to an exponential model and this inevitably introduces some error. Polynomial fitting of data is more accurate and robust and, for this reason, it is expected that the correction of viscometric data using the proposed series solution (in which the $\mu$ vs. $\Theta$ relationship is expressed by polynomials) will be more accurate than a correction based on analytical solutions such as Eq. (46). Furthermore, the solutions derived in this study allow for consideration of a temperature-dependent thermal conductivity. Besides their use in rheometry for the correction of viscometric data, such solutions can be linked, as independent explicit modules, to models for the evolution of microstructure (for example in particulate composites, (Gadala-Maria and Acrivos, 1980; Leighton and Acrivos, 1987) during flow. Section 2 is an outline of the mathematical model, of the method of solution and of the series solutions obtained. Section 3 is concerned with the validation of the series solutions using existing analytical results and a numerical solution of the model equations. Finally Section 4 presents results concerning the distribution of temperature and shear rate in the fluid as well as formal corrections to be used in the Couette viscometer.

\section{The Mathematical Model}

We consider steady, incompressible flow in a Couette device shown in Fig. 1. This device consists of two concentric cylinders of radii $R$ and $\varepsilon R(\varepsilon<1)$, of which one is stationary and the other is rotating with constant angular velocity $\Omega$. When the ratio between

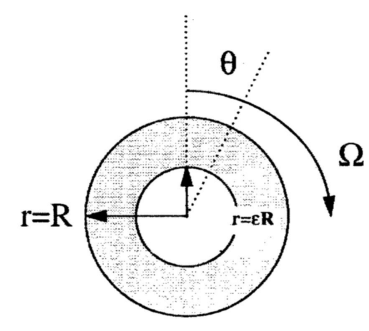

Fig. 1 Schematic description of the Couette apparatus

the diameters of the inner and the outer cylinders $(\varepsilon)$ approaches unity, the circular nature of the flow is progressively lost and it is reduced to flow between two infinite parallel plates moving with relative velocity $(V)$. This flow, termed planar-Couette flow, is of importance in the analysis of a range of rheological measurements. The only non-zero velocity component in this geometry is the velocity $(\phi)$ parallel to the moving plate, there is no pressure drop and the equations of motion and energy reduce to:

$$
\begin{aligned}
& \frac{\partial}{\partial z}\left\{\frac{\mu}{\mu_{0}} \cdot \frac{\partial u}{\partial z}\right\}=0 \\
& \frac{\partial}{\partial z}\left\{\frac{\kappa}{\kappa_{0}} \cdot \frac{\partial \Theta}{\partial z}\right\}+B r \cdot \frac{\mu}{\mu_{0}} \cdot\left[\frac{\partial u}{\partial z}\right]^{2}=0
\end{aligned}
$$

where the following dimensionless quantities have been used:

$$
\Theta=\frac{T-T_{0}}{T_{0}} \quad z=\frac{x}{L} \quad u=\frac{\phi}{V} \quad B r=\frac{\mu_{0} \cdot V^{2}}{\kappa_{0} \cdot T_{0}}
$$

In Eq. (3), $B r$ is the Brinkman number which is a measure of the heat generated by viscous heating as compared to the heat conducted through the material. $T_{0}$ is the temperature at the surfaces of the two plates, $(L)$ the height of the gap between the two plates and $(V)$ the velocity of the moving plate. Closed form solutions to Eqs. (1) - (2) have been obtained for certain limiting cases (Bird et al. 1960; Turian and Bird, 1963 and references therein; Gavis and Lawrence, 1968). In this work we are interested in obtaining approximate analytical solutions in series form to Eqs. (1) and (2) for fluids whose transport properties, namely the viscosity and the thermal conductivity, are arbitrary polynomial functions of temperature. Such dependencies can be expressed around a reference temperature $T_{0}$ as:

$$
\begin{aligned}
& \frac{\kappa}{\kappa_{0}}=1+\sum_{i=1}^{I} \alpha_{i} \cdot \Theta^{i} \\
& \frac{\mu_{0}}{\mu}=1+\sum_{i=1}^{I} \beta_{i} \cdot \Theta^{i}
\end{aligned}
$$


where the order of the approximations are not necessarily equal and where, in practice, the coefficients $\alpha_{i}$ and $\beta_{i}$ will be determined by fitting experimental data. This presentation of material property data is very common in the process industries and has been a motivation for the development of the solutions presented in this study. The subscript (0) in Eqs. (4) and (5) indicates properties evaluated at the reference temperature $T_{0}$. We consider the following boundary conditions:

$$
\text { At } z=0 \text { (stationary surface): } u=0 \text { and } \Theta=0
$$

$$
\text { At } z=1 \text { (moving surface): } u=1 \text { and } \Theta=0
$$

It should be noted that the solution procedure presented in this work is quite general and can admit derivative as well as non-zero Dirichlet conditions on either of the cylinder surfaces.

\subsection{Method of solution}

We seek approximate analytical solutions to Eqs. (1) and (2) subject to boundary conditions 6-7, for fluids with temperature-sensitive transport properties described by Eqs. (4) - (5). Such solutions can be formulated as perturbations with respect to the Brinkman number ( $\mathrm{Br}$ ) (Turian and Bird, 1963; Bird et al., 1960) as follows:

$$
\begin{aligned}
& u(z)=u_{0}(z)+\sum_{n=1}^{N} u_{n}(z) \cdot B r^{n} \\
& \Theta(z)=\Theta_{0}(z)+\sum_{n=1}^{N} \Theta_{n}(z) \cdot B r^{n}
\end{aligned}
$$

In the absence of viscous heating $(B r=0)$ the system is isothermal and therefore $\Theta_{0}(z)$ in Eq. (9) is identically zero. The objective of the solution procedure is to determine the coefficient functions $u_{n}(z)$ and $\Theta_{n}(z)$. This is outlined in the following two sections.

\subsubsection{The momentum equation Substitution of} the velocity profile from Eq. (8) into the momentum equation (Eq. (1)), yields:

$$
\frac{\partial}{\partial z}\left[\frac{\mu}{\mu_{0}} \cdot\left(\sum_{n=0}^{N} B r^{n} \cdot \frac{\partial u_{n}(z)}{\partial z}\right)\right]=0
$$

Integrating Eq. (10) once and expanding the integration constant $(C)$ as a polynomial in $(B r)$ yields:

$$
\begin{aligned}
\sum_{n=0}^{N} B r^{n} \cdot \frac{\partial u_{n}(z)}{\partial z} & =\frac{\mu_{0}}{\mu} \sum_{m=0}^{N} C_{m} \cdot B r^{m} \\
& =\left(1+\sum_{i=1}^{I} \beta_{i} \cdot \Theta^{i}\right) \cdot \sum_{m=0}^{N} C_{m} \cdot B r^{m}
\end{aligned}
$$

where the coefficients $C_{0}, C_{1}, \ldots C_{m}$ are constants to be determined and where the dependence of viscosity on temperature was taken into account. Equating equal powers of $(\mathrm{Br})$ on the left- and right-hand sides of Eq. (11) results in $(N+1)$ ordinary differential equations for the $(N+1)$ functions $u_{n}(z)$. The first five of these equations, corresponding to a solution of fourth order in $(B r)$, follow:

$$
\frac{\partial u_{0}}{\partial z}=C_{0}
$$

$$
\frac{\partial u_{1}(z)}{\partial z}=C_{1}+C_{0} \cdot \beta_{1} \cdot \Theta_{1}(z)
$$

$$
\begin{aligned}
\frac{\partial u_{2}(z)}{\partial z}= & C_{2}+C_{1} \cdot \beta_{1} \cdot \Theta_{1}(z) \\
& +C_{0} \cdot\left[\beta_{1} \cdot \Theta_{2}(z)+\beta_{2} \cdot \Theta_{1}{ }^{2}(z)\right] \\
\frac{\partial u_{3}(z)}{\partial z}= & C_{3}+C_{2} \cdot \beta_{1} \cdot \Theta_{1}(z) \\
& +C_{1} \cdot\left[\beta_{1} \cdot \Theta_{2}(z)+\beta_{2} \cdot \Theta_{1}{ }^{2}(z)\right] \\
& +C_{0} \cdot\left[\beta_{1} \cdot \Theta_{3}(z)+2 \beta_{2} \cdot \Theta_{1}(z) \Theta_{2}(z)\right. \\
& \left.+\beta_{3} \cdot \Theta_{1}{ }^{3}(z)\right]
\end{aligned}
$$

$$
\begin{aligned}
\frac{\partial u_{4}}{\partial z}=C_{0} & \cdot\left[\beta_{4} \Theta_{1}{ }^{4}+\beta_{2} \Theta_{2}{ }^{2}+\beta_{1} \Theta_{4}+3 \beta_{3} \Theta_{2} \Theta_{1}{ }^{2}\right. \\
& \left.+2 \beta_{2} \Theta_{3} \Theta_{1}\right]+C_{1} \cdot\left[\beta_{1} \Theta_{3}+\beta_{3} \Theta_{1}{ }^{3}\right. \\
& \left.+2 \beta_{2} \Theta_{2} \Theta_{1}\right]+\ldots \ldots \ldots . . \\
& +C_{2} \cdot\left[\beta_{1} \Theta_{2}+\beta_{2} \Theta_{1}{ }^{2}\right]+C_{3} \cdot \beta_{1} \Theta_{1}+C_{4}
\end{aligned}
$$

1.1.2 The energy equation It has been shown that a first integration of the momentum equation yields:

$$
\frac{\partial u}{\partial z}=\frac{\mu_{0}}{\mu} \sum_{m=0}^{N} C_{m} \cdot B r^{m}
$$

Substituting this result into the energy equation yields:

$$
\frac{\partial}{\partial z}\left(\frac{\kappa}{\kappa_{0}} \cdot \frac{\partial \Theta}{\partial z}\right)+B r \cdot \frac{\mu_{0}}{\mu} \cdot\left[\sum_{m=0}^{N} C_{m} \cdot B r^{m}\right]^{2}=0
$$

Taking into account the temperature dependence of $(\kappa)$ and $(\mu)$ and expressing $(\Theta)$ as a power-series in $(B r)$ as in Eq. (11), one gets:

$$
\begin{aligned}
& \frac{\partial}{\partial z}\left[\left(1+\sum_{i=1}^{I} \alpha_{i} \Theta(z)^{i}\right) \cdot \sum_{n=1}^{N} B r^{n} \cdot \frac{\partial \Theta_{n}(z)}{\partial z}\right] \\
& \quad+B r \cdot\left(1+\sum_{i=1}^{I} \beta_{i} \Theta(z)^{i}\right) \cdot\left[\sum_{m=0}^{N} C_{m} \cdot B r^{m}\right]^{2}=0
\end{aligned}
$$

As before, equating the coefficients of equal powers of $(B r)$ results in $(N)$ differential equations for the $(N)$ 
functions $\Theta_{n}(z)$. The first four of these, corresponding to a solution of second order in $(B r)$, follow:

$$
\begin{aligned}
& \frac{\partial}{\partial z}\left[\frac{\partial \Theta_{1}}{\partial z}\right]=-C_{0}^{2} \\
& \frac{\partial}{\partial z}\left[\alpha_{1} \Theta_{1}(z) \frac{\partial \Theta_{1}}{\partial z}+\frac{\partial \Theta_{2}}{\partial z}\right] \\
& =-C_{0} \cdot\left[C_{0} \beta_{1} \Theta_{1}(z)+2 C_{1}\right] \\
& \frac{\partial}{\partial z}\left[\alpha_{2} \Theta_{1}^{2}(z) \frac{\partial \Theta_{1}}{\partial z}+\alpha_{1} \Theta_{1}(z) \frac{\partial \Theta_{2}}{\partial z}\right. \\
& \left.\quad+\alpha_{1} \Theta_{2}(z) \frac{\partial \Theta_{1}}{\partial z}+\frac{\partial \Theta_{3}}{\partial z}\right] \\
& \quad=-C_{0}^{2} \cdot\left[\beta_{1} \Theta_{2}(z)+\beta_{2} \Theta_{1}^{2}(z)\right] \\
& \quad \ldots \ldots \ldots . .-2 C_{0} C_{1} \beta_{1} \Theta_{1}(z)-2 C_{0} C_{2}-C_{1}{ }^{2}
\end{aligned}
$$

$$
\begin{aligned}
\frac{\partial}{\partial z}[ & \left(\alpha_{2} \Theta_{1}^{2}+\alpha_{1} \Theta_{2}\right) \cdot \frac{\partial \Theta_{2}}{\partial z} \\
& +\left(2 \alpha_{2} \Theta_{1} \Theta_{2}+\alpha_{1} \Theta_{3}+\alpha_{3} \Theta_{1}^{3}\right) \cdot \frac{\partial \Theta_{1}}{\partial z} \\
& \left.+\alpha_{1} \Theta_{1} \cdot \frac{\partial \Theta_{3}}{\partial z}+\frac{\partial \Theta_{4}}{\partial z}\right]=\ldots \ldots \ldots \ldots . . . \\
& -C_{0}^{2} \cdot\left[\beta_{1} \Theta_{3}+\beta_{3} \Theta_{1}^{3}+2 \beta_{2} \Theta_{1} \Theta_{2}\right] \\
& -2 C_{0} C_{1} \cdot\left[\beta_{1} \Theta_{2}+\beta_{2} \Theta_{1}^{2}\right]+\ldots \ldots \ldots \ldots . . . \\
& -\left(2 C_{0} C_{2}+C_{1}^{2}\right) \cdot \beta_{1} \Theta_{1}-2 C_{0} C_{3}-2 C_{1} C_{2}
\end{aligned}
$$

A fourth-order in $(\mathrm{Br})$ solution for the planar Couette flow has been obtained in this work through analytical integration of the $(2 N+1=9)$ differential equations given by Eqs. (12) - (16) and (20) - (23). This solution for the velocity and temperature profiles follow:

\section{Velocity:}

$$
\begin{aligned}
u_{0}(z)= & z \\
u_{1}(z)= & -\frac{\beta_{1}}{6} \cdot z^{3}+\frac{\beta_{1}}{4} \cdot z^{2}-\frac{\beta_{1}}{12} \cdot z \\
u_{2}(z)= & \frac{1}{5} \cdot\left(\frac{1}{24} \cdot \beta_{1}^{2}-\frac{1}{8} \cdot \beta_{1} \cdot \alpha_{1}+\frac{1}{4} \cdot \beta_{2}\right) \cdot z^{5} \\
& +\frac{1}{4} \cdot\left(\frac{-1}{2} \cdot \beta_{2}-\frac{1}{12} \cdot \beta_{1}{ }^{2}+\frac{1}{4} \cdot \beta_{1} \cdot \alpha_{1}\right) \cdot z^{4} \ldots \\
& +\frac{1}{3} \cdot\left(\frac{1}{8} \cdot \beta_{1}{ }^{2}-\frac{1}{8} \cdot \beta_{1} \cdot \alpha_{1}+\frac{1}{4} \cdot \beta_{2}\right) \cdot z^{3} \\
& -\frac{1}{24} \cdot \beta_{1}{ }^{2} \cdot z^{2}+\left(\frac{1}{80} \cdot \beta_{1}{ }^{2}\right.
\end{aligned}
$$

$$
\left.+\frac{1}{240} \cdot \beta_{1} \cdot \alpha_{1}-\frac{1}{120} \cdot \beta_{2}\right) \cdot z
$$

$u_{3}(z)=\left[\frac{-1}{140} \cdot \beta_{1} \cdot \beta_{2}+\frac{1}{56} \cdot \beta_{2} \cdot \alpha_{1}+\frac{1}{168} \cdot \beta_{1} \cdot \alpha_{2}\right.$

$$
\begin{aligned}
& +\frac{1}{280} \cdot \beta_{1}^{2} \cdot \alpha_{1}-\frac{1}{112} \cdot \beta_{1} \cdot \alpha_{1}{ }^{2} \\
& \left.-\frac{1}{56} \cdot \beta_{3}-\frac{1}{5040} \cdot \beta_{1}^{3}\right) \cdot z^{7} \ldots
\end{aligned}
$$$$
+\left(\frac{1}{1440} \cdot \beta_{1}^{3}-\frac{1}{48} \cdot \beta_{1} \cdot \alpha_{2}+\frac{1}{40} \cdot \beta_{1} \cdot \beta_{2}\right.
$$$$
+\frac{1}{16} \cdot \beta_{3}-\frac{1}{16} \cdot \beta_{2} \cdot \alpha_{1}-\frac{1}{80} \cdot \beta_{1}^{2} \cdot \alpha_{1}
$$$$
\left.+\frac{1}{32} \cdot \beta_{1} \cdot \alpha_{1}^{2}\right) \cdot z^{6} \ldots
$$$$
+\left(\frac{1}{48} \cdot \beta_{1}^{2} \cdot \alpha_{1}-\frac{3}{80} \cdot \beta_{1} \cdot \alpha_{1}^{2}-\frac{1}{24} \cdot \beta_{1} \cdot \beta_{2}\right.
$$$$
+\frac{1}{40} \cdot \beta_{1} \cdot \alpha_{2}+\frac{3}{40} \cdot \beta_{2} \cdot \alpha_{1}-\frac{1}{288} \cdot \beta_{1}{ }^{3}
$$$$
\left.-\frac{3}{40} \cdot \beta_{3}\right) \cdot z^{5} \ldots
$$$$
+\left(\frac{1}{24} \cdot \beta_{1} \cdot \beta_{2}-\frac{1}{32} \cdot \beta_{2} \cdot \alpha_{1}+\frac{1}{144} \cdot \beta_{1}{ }^{3}\right.
$$$$
+\frac{1}{32} \cdot \beta_{3}-\frac{1}{48} \cdot \beta_{1}^{2} \cdot \alpha_{1}-\frac{1}{96} \cdot \beta_{1} \cdot \alpha_{2}
$$$$
\left.+\frac{1}{64} \cdot \beta_{1} \cdot \alpha_{1}^{2}\right) \cdot z^{4} \ldots
$$

$$
+\left(\frac{-1}{60} \cdot \beta_{1} \cdot \beta_{2}+\frac{1}{120} \cdot \beta_{1}^{2} \cdot \alpha_{1}\right.
$$$$
\left.-\frac{7}{720} \cdot \beta_{1}{ }^{3}\right) \cdot z^{3}+\left(\frac{-1}{240} \cdot \beta_{1} \cdot \beta_{2}\right.
$$$$
\left.+\frac{23}{2880} \cdot{\beta_{1}}^{3}+\frac{1}{480} \cdot \beta_{1}{ }^{2} \cdot \alpha_{1}\right) \cdot z^{2} \ldots
$$$$
+\left(\frac{\beta_{1} \cdot \beta_{2}}{336}+\frac{\beta_{2} \cdot \alpha_{1}}{1120}+\frac{\beta_{1} \cdot \alpha_{2}}{3360}\right.
$$$$
-\frac{1}{672} \cdot \beta_{1}{ }^{2} \cdot \alpha_{1}-\frac{1}{2240} \cdot \beta_{1} \cdot \alpha_{1}{ }^{2}
$$$$
\left.-\frac{1}{1120} \cdot \beta_{3}-\frac{1}{448} \cdot \beta_{1}{ }^{3}\right) \cdot z
$$ 


$$
\begin{aligned}
u_{4}(z)= & \frac{\Delta_{9}}{9} \cdot z^{9}+\frac{\Delta_{8}}{8} \cdot z^{8}+\frac{\Delta_{7}}{7} \cdot z^{7}+\frac{\Delta_{6}}{6} \cdot z^{6} \\
& +\frac{\Delta_{5}}{5} \cdot z^{5}+\frac{\Delta_{4}}{4} \cdot z^{4}+\frac{\Delta_{3}}{3} \cdot z^{3}+\frac{\Delta_{2}}{2} \cdot z^{2} \\
& +\Delta_{1} \cdot z
\end{aligned}
$$

where the coefficients $\Delta_{i}, i=1,9$ are given in Appendix A.

\section{Temperature:}

$$
\begin{aligned}
\Theta_{1}(z)= & z \cdot \frac{(1-z)}{2} \\
\Theta_{2}(z)= & \left(\frac{1}{24} \cdot \beta_{1}-\frac{1}{8} \cdot \alpha_{1}\right) \cdot z^{4} \\
& +\left(\frac{-1}{12} \cdot \beta_{1}+\frac{1}{4} \cdot \alpha_{1}\right) \cdot z^{3} \\
& +\left(\frac{1}{12} \cdot \beta_{1}-\frac{1}{8} \cdot \alpha_{1}\right) \cdot z^{2}-\frac{1}{24} \cdot \beta_{1} \cdot z
\end{aligned}
$$

$$
\Theta_{3}(z)=\left(\frac{-1}{720} \cdot \beta_{1}{ }^{2}-\frac{1}{120} \cdot \beta_{2}+\frac{1}{40} \cdot \beta_{1} \cdot \alpha_{1}\right.
$$$$
\left.+\frac{1}{24} \cdot \alpha_{2}-\frac{1}{16} \cdot \alpha_{1}^{2}\right) \cdot z^{6} \ldots
$$$$
+\left(\frac{-3}{40} \cdot \beta_{1} \cdot \alpha_{1}+\frac{1}{40} \cdot \beta_{2}+\frac{3}{16} \cdot \alpha_{1}^{2}\right.
$$$$
\left.-\frac{1}{8} \cdot \alpha_{2}+\frac{1}{240} \cdot \beta_{1}^{2}\right) \cdot z^{5} \ldots
$$$$
+\left(\frac{-1}{72} \cdot \beta_{1}{ }^{2}+\frac{3}{32} \cdot \beta_{1} \cdot \alpha_{1}-\frac{3}{16} \cdot \alpha_{1}{ }^{2}\right.
$$$$
\left.-\frac{1}{48} \cdot \beta_{2}+\frac{1}{8} \cdot \alpha_{2}\right) \cdot z^{4} \ldots
$$$$
+\left(\frac{1}{48} \cdot \beta_{1}^{2}-\frac{1}{16} \cdot \beta_{1} \cdot \alpha_{1}+\frac{1}{16} \cdot \alpha_{1}^{2}\right.
$$$$
\left.-\frac{1}{24} \cdot \alpha_{2}\right) \cdot z^{3} \ldots
$$$$
+\left(\frac{-23}{1440} \cdot \beta_{1}^{2}+\frac{1}{120} \cdot \beta_{2}+\frac{1}{60} \cdot \beta_{1} \cdot \alpha_{1}\right) \cdot z^{2}
$$$$
+\left(\frac{1}{160} \cdot \beta_{1}{ }^{2}-\frac{1}{240} \cdot \beta_{2}+\frac{1}{480} \cdot \beta_{1} \cdot \alpha_{1}\right) \cdot z
$$

$$
\begin{aligned}
\Theta_{4}(z)= & \Gamma_{8} \cdot z^{8}+\Gamma_{7} \cdot z^{7}+\Gamma_{6} \cdot z^{6}+\Gamma_{5} \cdot z^{5}+\Gamma_{4} \cdot z^{4} \\
& +\Gamma_{3} \cdot z^{3}+\Gamma_{2} \cdot z^{2}+\Gamma_{1} z
\end{aligned}
$$

where the coefficients $\Gamma_{i}, i=1,8$ are given in Appendix B:

\section{Validation of the Series Solutions}

\subsection{Comparison with existing approximate and exact solutions}

It can be seen that the leading terms of the presented series solutions $\left(u_{0}\right.$ and $\left.\Theta_{1}\right)$ correspond, as expected, to existing analytical results (Bird et al., 1960). In the following we compare the proposed 4th-order series solution with the predictions of a closed-form analytical solution for planar Couette flow for a material with constant thermal conductivity $\left(\alpha_{\mathrm{n}}=0, n=1, N\right)$ and viscosity-temperature dependence described by an exponential decay $\left(\mu=\mu_{0}\right.$.exp $(-\lambda \Theta))$. In this case, an exact analytical solution to the problem posed by Eqs. (1) - (2) exists (Nahme, 1940; Turian and Bird, 1963):

$$
\begin{aligned}
u_{\mathrm{ex}}(z)= & \frac{1}{2} \cdot\left[1+\left(1+\frac{8}{B r \cdot \lambda}\right)^{1 / 2}\right. \\
& \left.\times \tanh \left\{(2 z-1) \cdot \arcsin h \sqrt{\frac{B r \cdot \lambda}{8}}\right\}\right] \\
\exp \left(\lambda \cdot \Theta_{\mathrm{ex}}(z)\right)= & \left(1+\frac{8}{B r \cdot \lambda}\right) \\
& \left.\times \sec h^{2}\left\{(2 z-1) \cdot \arcsin h \sqrt{\frac{B r \cdot \lambda}{8}}\right\}\right]
\end{aligned}
$$

Use of the product $B r \lambda$, which appears in Eqs. (33) (34), is necessary to quantify the effect of viscous heating on the gap-wise velocity profile. For this purpose, quoting the $\mathrm{Br}$ alone is not sufficient since the degree of deviation from simple shear flow depends not only on the degree of viscous heating (which is expressed by $B r$ ) but also on the sensitivity of the fluid viscosity on temperature (which is expressed by $\lambda$ ). The correspondence between $(\lambda)$ and the coefficients $\beta_{i}$ in Eq.(5) is obtained by expanding $\exp (\lambda \Theta)$ as a Taylor series:

$$
\beta_{1}=\lambda \quad \beta_{2}=\frac{\lambda^{2}}{2} \quad \beta_{3}=\frac{\lambda^{3}}{6} \quad \beta_{4}=\frac{\lambda^{4}}{4 !}
$$

A comparison between Eqs. (33) and (34) and the predictions of the 4th-order series solution (Eqs. (24) (32)) for this limiting case is shown in Fig. 2, for $B r \lambda=5$. In the presence of a temperature-dependent viscosity, the velocity profile becomes distinctively $\mathrm{S}$ shaped and the flow field deviates significantly from simple shear as $B r$ is increased. It is evident that at 


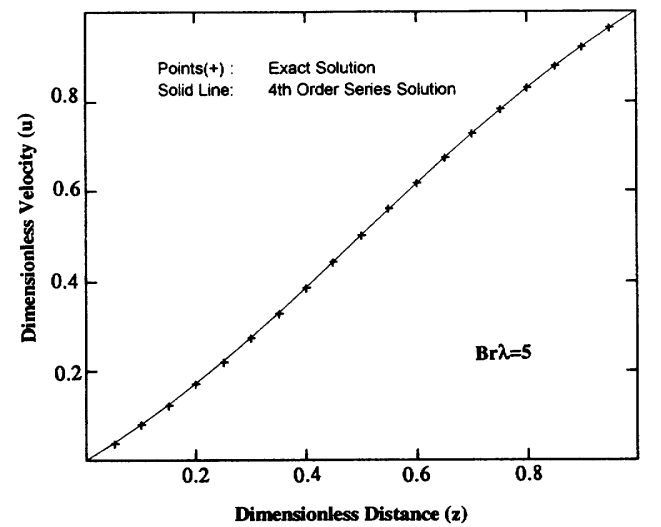

Fig. 2 Predictions of the 4th-order series solution and of the exact solution for the velocity profile in planar Couette flow, in the case of a fluid with constant thermal conductivity $\left(\alpha_{1}=\alpha_{2}=\alpha_{3}=0\right)$ and exponential viscosity-temperature dependence $\left(\beta_{1}=\lambda=\right.$ $2, \beta_{2}=2, \beta_{3}=1.333, \beta_{4}=0$ )

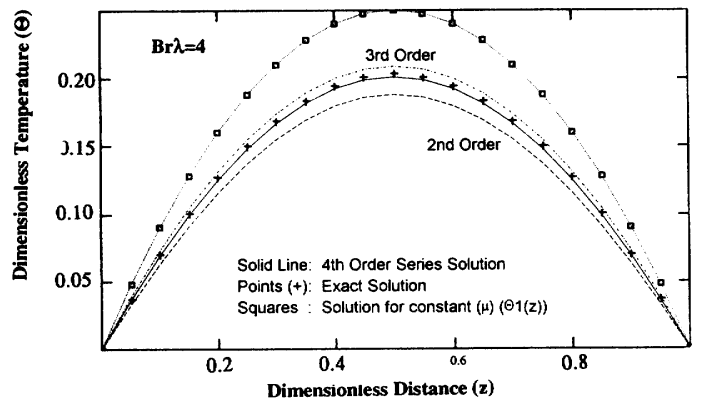

Fig. 3 Predictions of series solutions of 2 nd, 3 rd and 4th order in $\mathrm{Br}$ and of the exact solution for the temperature profile, in planar Couette flow of a fluid with constant thermal conductivity $\left(\alpha_{1}=\alpha_{2}=\right.$ $\left.\alpha_{3}=0\right)$ and exponential viscosity-temperature dependence $\left(\beta_{1}=\lambda=2, \beta_{2}=2, \beta_{3}=1.333, \beta_{4}=0\right)$

least up to $B r \lambda=5$ the series and analytical solutions are practically indistinguishable. The temperature profiles obtained for $B r \lambda=4$ are shown in Fig. 3, in which the temperature profile corresponding to a fluid with constant transport properties $\left(\Theta_{1}(z)\right.$-Eq. (29)) is also shown. Evidently, ignoring the temperature effects on $(\mu)$ and $(\kappa)$ results in a significant overestimation of the temperature rise caused by viscous dissipation; the first-order result $\Theta_{1}(z)$ can be considered a conservative estimate for over-heating. It is also evident that the 4th-order solution is significantly more accurate than the 2nd- and 3rdorder solutions. An appreciation of the improvement offered by increasing the order of the series approximation is shown in Figs. 4a-c, in which the difference between the series and the exact solution for the velocity profile, namely the quantity $[u(z)-$ $\left.u_{\text {ex }}(z)\right]^{*} 100$ where $u_{\text {ex }}(z)$ is given by Eq. (33), is plotted across the gap of the Couette for three values of the
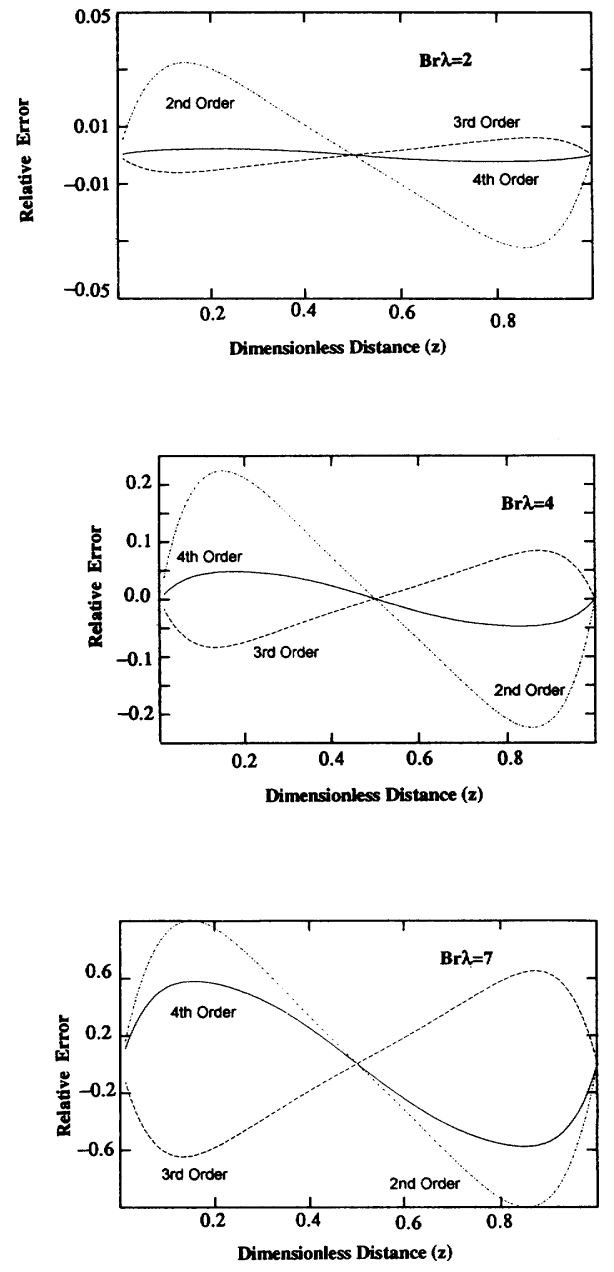

Fig. 4 Effect of the order of the series solution for the velocity profile in planar Couette flow on its deviation from the exact solution. Plotted is the quantity $\left[u(z)-u_{\mathrm{ex}}(z)\right]^{*} 100$ where $u(z)$ is given by Eqs. (24) $-(28)$ and $u_{\text {ex }}(z)$ by Eq. (33) for three values of the Brinkman number. $\left(\alpha_{1}=\alpha_{2}=\alpha_{3}=0\right.$; $\beta_{n}=\left(\beta^{n}\right) / n$ !) Evidently, increasing the order of approximation improves the accuracy of the series solution

product $B r \lambda$. Evidently, increasing the order of the series solution improves its accuracy-or, equivalently, extends the range of its validity to higher values of $B r$. Solutions of higher order in $(B r)$ allow for inclusion of higher order terms in the polynomial approximation of the viscosity-temperature curve. This allows for accurate approximation of the latter over a wider range of temperatures, thus making the series solution applicable to higher values of $B r \lambda$.

\subsection{Comparison with numerical solution}

The comparison with the exact solution presented previously is only a partial test of the validity of the series solution derived in this study. The analytical solution assumes that the viscosity is an exponential function of temperature, while the series 
Table 1 The norm of the difference between numerical and series solutions of the second, third and fourth orders, for a range of values of the Brinkman number $\beta_{1}=\lambda=1, \beta_{\mathrm{n}}=\left(\beta_{1}{ }^{\mathrm{n}}\right) / n !(n=2,3,4) \alpha_{1}=0.5, \alpha_{2}=0.4, \alpha_{3}=0.15$

\begin{tabular}{|c|c|c|c|c|c|c|}
\hline \multirow{2}{*}{$B r \lambda$} & \multicolumn{3}{|c|}{ Norm $\left(u_{R}\right)$} & \multicolumn{3}{|c|}{$\operatorname{Norm}\left(\Theta_{R}\right)$} \\
\hline & 2nd-order & 3 rd-order & 4th-order & 2nd-order & 3rd-order & 4th-order \\
\hline 1.0 & 0.0248 & 0.0066 & 0.0019 & 1.011 & 0.2176 & 0.0470 \\
\hline 2.0 & 0.1858 & 0.0749 & 0.0071 & 3.923 & 1.378 & 0.1641 \\
\hline 2.5 & 0.3521 & 0.1658 & 0.0294 & 6.041 & 2.532 & 0.5838 \\
\hline 3.0 & 0.5908 & 0.3183 & 0.0930 & 8.578 & 4.179 & 1.3838 \\
\hline 3.5 & 0.9122 & 0.5532 & 0.2203 & 11.521 & 6.394 & 2.7152 \\
\hline 4.0 & 1.325 & 0.894 & 0.445 & 14.859 & 9.253 & 4.755 \\
\hline 4.5 & 1.837 & 1.364 & 0.8096 & 18.581 & 12.825 & 7.695 \\
\hline 5.0 & 2.457 & 1.991 & 1.365 & 22.677 & 17.182 & 11.747 \\
\hline
\end{tabular}

solution considers the more general situation of polynomial dependence. Since the 4 th-order polynomial expansion will inevitably deviate from the exponential at large $\Theta$, it becomes evident that, for "large" $\Theta$ the analytical solution of Eq. $(33-34)$ and the presented series solution are not comparable. Furthermore, the analytical solution assumes that the thermal conductivity of the fluid is constant, while the series solution allows for a cubic dependence on temperature. In order to determine the range of validity of the proposed series solution at higher values of overheating as well as for non-constant thermal conductivity, the boundary-value problem defined by Eqs. (1) - (2) is solved numerically, subject to the boundary conditions of Eqs. $(6-7)$. The material properties used are given by Eqs. (4) and (5), with $\lambda=1$ for the viscosity model and $\alpha_{1}=0.5, \alpha_{2}=0.4, \alpha_{3}=0.15$ for the thermal conductivity model. The latter predicts an increase of $(\kappa)$ with $(\Theta)$, in qualitative agreement with results on the thermal conductivity of food-related products (Halliday and Smith, 1993). A more conventional dependence of $(\kappa)$ on temperature $[(\partial \kappa / \partial \Theta)<0]$ can be considered by proper selection of $\alpha_{1}, \alpha_{2}, \alpha_{3}$.

The governing equations (1) - (2) are rendered explicit in the gradients of $(u)$ and $(\Theta)$ through application of the chain-rule. The momentum equation takes the form:

$$
\mu(z) \frac{\partial^{2} u(z)}{\partial^{2} z}+A(z) \frac{\partial u(z)}{\partial z}=0
$$

with

$$
A(z)=-\frac{\begin{array}{c}
{\left[\beta_{1}+2 \beta_{2} \Theta(z)+3 \beta_{3} \Theta^{2}(z)\right.} \\
\left.+4 \beta_{4} \Theta^{3}(z)\right]
\end{array}}{\begin{array}{c}
{\left[1+\beta_{1} \Theta(z)+\beta_{2} \Theta^{2}(z)\right.} \\
\left.+\beta_{3} \Theta^{3}(z)+\beta_{4} \Theta^{4}(z)\right]^{2}
\end{array}} \cdot \frac{\partial \Theta(z)}{\partial z}
$$

while the energy equation can be written as:

$$
\begin{gathered}
\frac{\kappa(z)}{\kappa_{0}} \frac{\partial^{2} \Theta(z)}{\partial z^{2}}+B(z) \frac{\partial \Theta(z)}{\partial z} \\
+B r \frac{\mu(z)}{\mu_{0}}\left(\frac{\partial u(z)}{\partial z}\right)^{2}=0
\end{gathered}
$$

where

$$
B(z)=\left[\alpha_{1}+2 \alpha_{2} \Theta(z)+3 \alpha_{3} \Theta^{2}(z)\right] \cdot \frac{\partial \Theta(z)}{\partial z}
$$

Equations (36) and (38) are discretised using central finite differences and solved using a successive relaxation algorithm (Press et al., 1985). The accuracy of the numerical algorithm has been verified through detailed comparison with the analytical solution of Eqs. (33) - (34) $\left(\alpha_{i}=0, i=1,3\right)$.

Table 1 summarises the results of a comparison between numerical (based on a 400-node spatial discretisation) and series solutions of $2 \mathrm{nd}$, 3rd and 4th order, for values of $B r \lambda$ ranging from 1 to 5 . Listed are the values of the norm of the percent relative differences of velocity and temperature $\operatorname{norm}\left(u_{R}\right)$ and $\operatorname{norm}\left(\Theta_{R}\right)$ respectively), defined as:

$$
\begin{aligned}
& \operatorname{norm}\left(u_{R}\right)=\frac{1}{K} \times 100 \times \sum_{j=1}^{K}\left|\frac{u_{s}-u_{a n}}{u_{a n}}\right|_{j} \\
& \text { and } \operatorname{norm}\left(\Theta_{R}\right)=\frac{1}{K} \times 100 \times \sum_{j=1}^{K}\left|\frac{\Theta_{s}-\Theta_{a n}}{\Theta_{a n}}\right|_{j}
\end{aligned}
$$

where the subscripts $(s)$ and (an) indicate series and numerical solutions respectively, the subscript $(j)$ indicates solution at the $j^{\text {th }}$ node and $(K)$ is the number of uniformly-spaced nodes across the gap at which the solution is evaluated.

Evidently, the accuracy of the new third- and fourth-order series solutions is quite satisfactory for an extended range of $B r \lambda$, and is a definite improvement over the second-order solution of Turian and Bird (1963). In particular, the norm of the errors corresponding to the third-order velocity profile is less 
Table 2 Comparison between numerical $\left(u_{\text {num }}, \Theta_{\text {num }}\right)$ and series solutions for temperature and velocity across the gap of a wide-gap Couette for $B r \lambda=3.0 \beta_{1}=\lambda=1, \beta_{\mathrm{n}}=\left(\beta_{1}{ }^{\mathrm{n}}\right) / n !(n=2,3,4) \alpha_{1}=0.5, \alpha_{2}=0.4, \alpha_{3}=0.15$

\begin{tabular}{|c|c|c|c|c|c|c|c|c|}
\hline \multirow{2}{*}{$z$} & \multicolumn{4}{|c|}{ Velocity } & \multicolumn{4}{|c|}{ Temperature } \\
\hline & $u_{\text {num }}$ & 2nd-order & 3rd-order & 4 th-order & $\Theta_{\text {num }}$ & 2nd-order & 3rd-order & 4th-order \\
\hline 0.099 & 0.0858 & 0.0875 & 0.0850 & 0.0861 & 0.1083 & 0.0991 & 0.1121 & 0.1065 \\
\hline 0.198 & 0.1802 & 0.1821 & 0.1792 & 0.1805 & 0.1907 & 0.1742 & 0.1983 & 0.1880 \\
\hline 0.298 & 0.2814 & 0.2829 & 0.2805 & 0.2816 & 0.2487 & 0.2270 & 0.2596 & 0.2454 \\
\hline 0.397 & 0.3874 & 0.3882 & 0.3869 & 0.3875 & 0.2834 & 0.2586 & 0.2966 & 0.2798 \\
\hline 0.496 & 0.4959 & 0.4959 & 0.4959 & 0.4959 & 0.2954 & 0.2695 & 0.3095 & 0.2917 \\
\hline 0.595 & 0.6045 & 0.6038 & 0.6050 & 0.6044 & 0.2850 & 0.2601 & 0.2984 & 0.2814 \\
\hline 0.695 & 0.7108 & 0.7093 & 0.7116 & 0.7106 & 0.2521 & 0.2301 & 0.2632 & 0.2488 \\
\hline 0.794 & 0.8124 & 0.8106 & 0.8135 & 0.8122 & 0.1958 & 0.1790 & 0.2037 & 0.1931 \\
\hline 0.893 & 0.9074 & 0.9057 & 0.9083 & 0.9071 & 0.1152 & 0.1055 & 0.1195 & 0.1135 \\
\hline 0.993 & 0.9939 & 0.9937 & 0.9940 & 0.9938 & 0.0089 & 0.0083 & 0.0093 & 0.0089 \\
\hline
\end{tabular}

than $1 \%$ for $B r \lambda$ up to 4.5 . The same is not true for the temperature profile, with the norm of the errors being less than $5 \%$ only for $B r \lambda$ up to 4.0 . The maximum deviation between series and numerical solutions occurs at the centre. A detailed comparison between the numerical and the series solutions for $B r \lambda=3.0$ is given in Table 2 .

\section{Results and Discussion}

\subsection{Maximum temperature and shear rate}

One of the consequences of a temperaturedependent viscosity in narrow-gap Couette flow is the development of a non-homogeneous shear field. As shown in Fig. 2, the velocity profile becomes S-shaped and the shear-rate profile non-homogeneous, with a maximum at the centre of the gap and minimum values on the walls. Considering that planar Couette flow is applied in rheometry because of its "simplicity" (constant shear rate) it is of significant interest to assess the deviation from simple-shear brought about by viscous heating and temperature-dependent transport properties. The shear rate profile across the gap $(\gamma(z))$ can be readily calculated by differentiating the velocity profile (Eqs. (24)-(28)). The result is omitted here for the sake of brevity. Figure 5 shows the shear rate profile across the gap of the Couette for the same conditions as in Fig. 2 . The shear rate $\left(\gamma_{\mathrm{ex}}\right)$ obtained from the exact solution (Eq. (33)) and given by Eq. (41) is included for comparison.

$$
\begin{aligned}
\gamma_{\mathrm{ex}}(z) \equiv & \frac{d u_{\mathrm{ex}}(z)}{d z}=\left(1+\frac{8}{B r \lambda}\right)^{1 / 2} \\
& {\left[1-\tanh \left\{(2 z-1) \arcsin h\left(\frac{\sqrt{2 B r \lambda}}{4}\right)\right\}^{2}\right] } \\
& \arcsin h\left(\frac{\sqrt{2 B r \lambda}}{4}\right)
\end{aligned}
$$

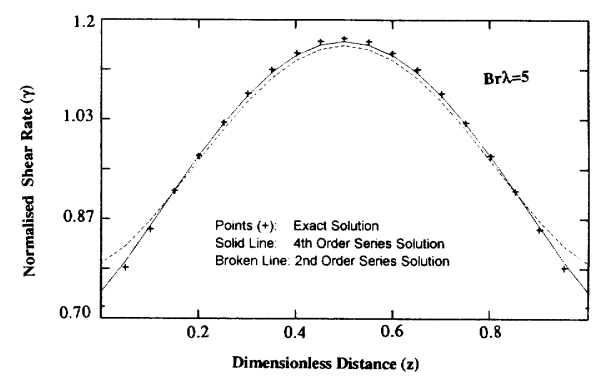

Fig. 5 Predictions of the series solution and of the exact solution for the shear-rate profile in planar Couette flow of a fluid with constant thermal conductivity $\left(\alpha_{1}=\alpha_{2}=\alpha_{3}=0\right)$ and exponential viscosity-temperature dependence $\left(\beta_{\mathrm{n}}=\left(\beta^{\mathrm{n}}\right) / n !\right)$

It is evident that even though both the second-and the fourth-order series solutions were able to accurately reproduce the velocity profile, the same cannot be said for the shear-rate profile, particularly so near the walls of the device where $(\gamma)$ attains its minimum value. It is seen that the 4th-order solution is significantly more accurate than the 2 nd-order one and therefore is expected to yield more accurate estimates concerning the interpretation of rheological results based on the measurement of forces exerted by the fluid on the rotating cylinder. For the boundary conditions expressed by Eqs. $(6-7)$ the maximum shear rate $\left(\gamma_{\max }\right)$ in the narrow-gap Couette occurs at $z=0.5$. The value of $\left(\gamma_{\max }\right)$ depends on the Brinkman number as well as on the sensitivity of the transport properties of the fluid on temperature (expressed by the coefficients $\alpha_{i}$ and $\beta_{i}$ in Eqs. (4) and (5)) and is given by:

$$
\gamma_{\max }=1+\frac{\beta_{1}}{24} B r+\gamma_{2} \cdot B r^{2}+\gamma_{3} \cdot B r^{3}+\gamma_{4} \cdot B r^{4}+\ldots
$$

where the coefficients $\gamma_{2}, \gamma_{3}$ and $\gamma_{4}$ are as follows: 


$$
\begin{aligned}
\gamma_{2}= & \frac{-11}{1920} \cdot \beta_{1}{ }^{2}-\frac{7}{1920} \cdot \beta_{1} \cdot \alpha_{1}+\frac{7}{960} \beta_{2} \\
\gamma_{3}= & \frac{-53}{26880} \cdot \beta_{1} \cdot \beta_{2}-\frac{19}{17920} \cdot \beta_{2} \cdot \alpha_{1} \\
& -\frac{19}{53760} \cdot \beta_{1} \cdot \alpha_{2}+\frac{53}{53760} \cdot \beta_{1}{ }^{2} \cdot \alpha_{1} \ldots \\
& +\frac{19}{35840} \cdot \beta_{1} \cdot \alpha_{1}{ }^{2}+\frac{19}{17920} \cdot \beta_{3}+\frac{103}{107520} \cdot \beta_{1}{ }^{3} \\
\gamma_{4}= & \sum_{n=1}^{9}\left[\Delta_{n} \cdot\left(\frac{1}{2}\right)^{n-1}\right]
\end{aligned}
$$

where the coefficients $\Delta_{n}, n=1,9$ come from the velocity profile (Eq. (28)) and are given in Appendix A.

Of interest in applications where viscous heating may be important is the prediction of the maximum temperature in the fluid. It can be shown that under the boundary conditions of Eqs. (6) - (7) the maximum temperature occurs at $z=0.5$. This maximum temperature is:

$$
\begin{aligned}
\Theta_{\max }= & B r \cdot \Theta_{\max }^{(1)}+B r^{2} \cdot \Theta_{\max }^{(2)} \\
& +B r^{3} \cdot \Theta_{\max }^{(3)}+B r^{4} \cdot \Theta_{\max }^{(4)}+\ldots
\end{aligned}
$$

where the coefficients $\Theta_{\max }^{(j)}, j=1 . .4$ depend on the properties of the fluid:

$$
\begin{aligned}
\Theta_{\max }^{(1)}= & \frac{1}{8} \\
\Theta_{\max }^{(2)}= & \frac{-1}{128} \cdot\left[\alpha_{1}+\beta_{1}\right] \\
\Theta_{\max }^{(3)}= & \frac{1}{1024} \cdot\left[\alpha_{1}{ }^{2}+\beta_{1}{ }^{2}\right]-\frac{1}{1536} \cdot\left[\alpha_{2}+\beta_{2}\right] \\
& +\frac{1}{768} \alpha_{1} \beta_{1} \\
\Theta_{\max }^{(4)}= & \frac{-25}{98304} \alpha_{1} \beta_{1}\left(\alpha_{1}+\beta_{1}\right)-\frac{5}{32768}\left(\alpha_{1}{ }^{3}+\beta_{1}{ }^{3}\right) \\
& -\frac{1}{16384}\left(\alpha_{3}+\beta_{3}\right)+\frac{7}{49152}\left(\alpha_{1} \beta_{2}+\alpha_{2} \beta_{1}\right) \\
& +\ldots \ldots \ldots . .+\frac{5}{24576}\left(\beta_{1} \beta_{2}+\alpha_{2} \alpha_{1}\right)
\end{aligned}
$$

The sensitivity of $\Theta_{\max }$ with respect to $B r$ as well as with respect to variations in the parameters $\alpha_{1}$ and $\beta_{1}$ is shown in Figs. 6a-c. Contrary to the second-order solution which predicts a linear dependence of $\Theta_{\max }$ on
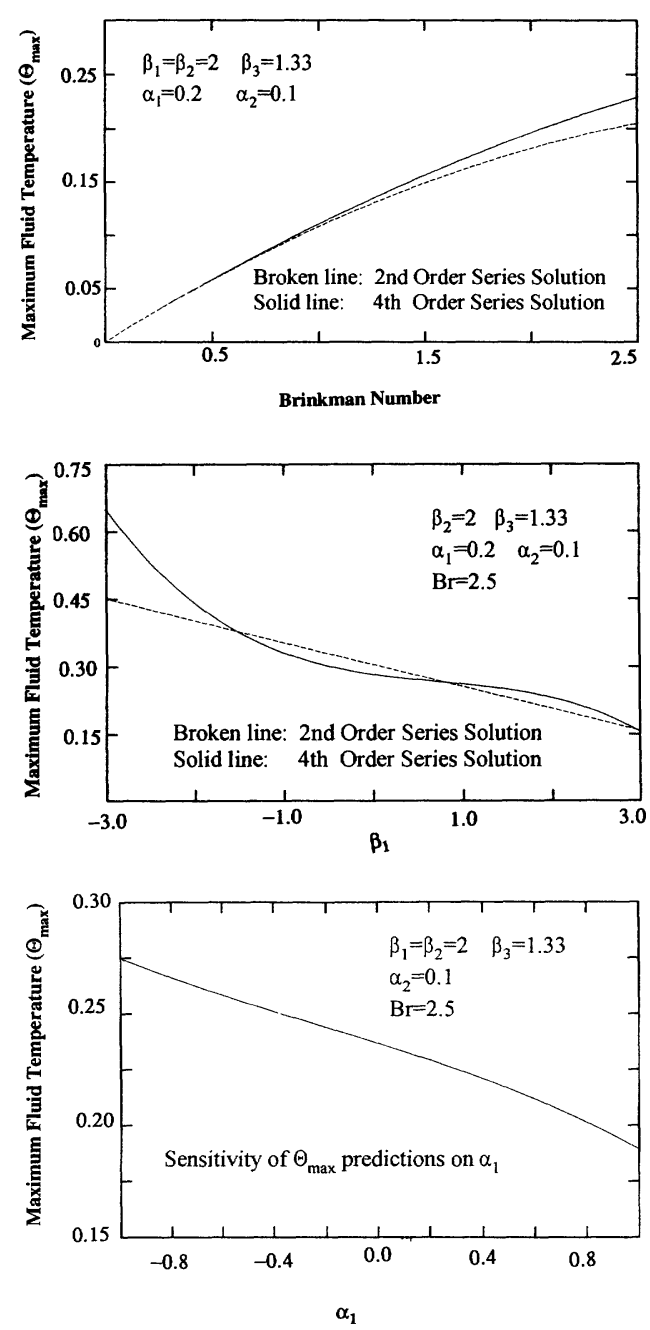

Fig. 6 Effect of $(\mathrm{Br})$ on the maximum temperature rise in planar Couette flow (a); Effect of the material constants $\beta_{1}$ and $\alpha_{1}$ on the predicted maximum temperature $(\mathrm{b}, \mathrm{c})$. Transport properties as shown in graphs

$\beta_{1}$, the more accurate 4 th-order solution predicts a more complex dependence and shows that $\Theta_{\max }$ is rather insensitive to variations in the value of the parameter $\beta_{1}$ when the latter varies between 0 and 1 (Fig. 6.b).

\subsection{Rheological corrections in a Couette viscometer}

The solutions derived and validated in this study can be used to develop corrections for the interpretation of angular velocity vs. torque data obtained during viscosity measurements through the use of the device described in Fig. 1, in cases where viscous heating and its effect on fluid properties are important. Elementary analysis shows that the torque $(F)$ required to turn the outer shaft of the viscometer is the product of the force exerted by the fluid on the outer (rotating) cylinder $(z=1)$ times the lever arm $(H)$ :

$$
F=2 \pi R^{2} H \mu_{0}(\gamma)_{z=1}
$$


Through Eq. (44), the viscosity of the fluid is found as the ratio between the normalised torque (measured experimentally) and the shear rate on the surface of the rotating cylinder. The latter is generally unknown and its determination requires knowledge of the velocity field between the two cylinders. When this is not known a-priori, the wall shear rate is calculated assuming simple shear flow. In this case, the viscosity measured through Eq. (44) is an "apparent" viscosity, which will only coincide with the true viscosity of the fluid in the absence of nonNewtonian or thermal effects. When such effects are present, the apparent viscosity needs to be corrected by considering the actual velocity profile. In the case of a Newtonian fluid with temperature-dependent transport properties, a solution for the velocity profile in the presence of viscous heating has been presented in this work, and therefore, Eq. (44) can be re-written as:

$$
\begin{aligned}
F= & 2 \pi R^{2} H \mu_{0}\left[1+B r \gamma_{1}^{(w)}+B r^{2} \gamma_{2}^{(w)}+B r^{3} \gamma_{3}^{(w)}\right. \\
& \left.+B r^{4} \gamma_{4}^{(w)}\right]
\end{aligned}
$$

where the coefficients $\gamma_{1}-\gamma_{4}$ can be found by evaluating the derivative of the velocity profile at the wall. It is a test for the validity of the presented 4th-order solution that this derivative assumes analytically the same value at $z=0$ and $z=1$. The coefficients $\gamma_{1}^{(w)}$ $\gamma_{4}{ }^{(w)}$ in Eq. (45) are:

$$
\begin{aligned}
& \gamma_{1}^{(w)}=-\frac{\beta_{1}}{12} \\
& \gamma_{2}^{(w)}=\frac{1}{80} \beta_{1}^{2}+\frac{1}{240} \beta_{1} \alpha_{1}-\frac{1}{120} \beta_{2} \\
& \gamma_{3}^{(w)}=\frac{1}{336} \beta_{1} \beta_{2}+\frac{1}{1120} \beta_{2} \alpha_{1}+\frac{1}{3360} \beta_{1} \alpha_{2} \\
& -\frac{1}{672} \beta_{1}^{2} \alpha_{1}-\frac{1}{2240} \beta_{1} \alpha_{1}^{2}-\frac{1}{1120} \beta_{3} \\
& -\frac{1}{448} \beta_{1}^{3} \\
& \gamma_{4}^{(w)}=\frac{1}{6720} \beta_{3} \alpha_{1}-\frac{1}{12096} \beta_{1} \alpha_{2} \alpha_{1}-\frac{1}{1152} \beta_{2} \beta_{1}{ }^{2} \\
& +\frac{1}{2304} \beta_{1}{ }^{3} \alpha_{1}+\frac{1}{2304} \beta_{1}{ }^{4}+\frac{1}{15120} \beta_{2} \alpha_{2} \\
& +\frac{1}{6048} \beta_{2}^{2}- \\
& \ldots \ldots \frac{1}{8064} \beta_{2} \alpha_{1}{ }^{2}-\frac{13}{24192} \beta_{1} \beta_{2} \alpha_{1}
\end{aligned}
$$

The bracketed term in Eq. (45) is usually termed the "correction term". It expresses the deviation of the actual wall shear rate from the one corresponding to simple shear flow and thus "corrects" the "apparent" viscosity calculated through the assumption of simple shear. Its magnitude is a function of the sensitivity of the transport properties of the fluid on temperature in the presence of viscous heating.

In the limiting case of constant thermal conductivity and exponential viscosity-temperature dependence, Nahme's solution (Eq. (33)) yields the following expression for the shear rate at the wall:

$$
\gamma_{w}=\frac{8}{\sqrt{B r \cdot \lambda(8+B r \cdot \lambda)}} \cdot \arcsin h\left(\frac{1}{4} \sqrt{2 B r \cdot \lambda}\right)
$$

This can be expanded as a Taylor series with respect to $(B r \cdot \lambda)$ as follows:

$$
\begin{aligned}
\gamma_{w}{ }^{(s)}= & 1-8.3333 \times 10^{-2} \cdot B r \cdot \lambda+8.3333 \\
& \times 10^{-3} \cdot(B r \cdot \lambda)^{2}-8.9286 \times 10^{-4} \cdot(B r \cdot \lambda)^{3}+ \\
& \ldots \ldots . .+9.9206 \times 10^{-5} \cdot(B r \cdot \lambda)^{4}+\ldots .
\end{aligned}
$$

It can be shown that the correction term in Eq. (45) corresponds exactly to the above series expansion, when $\alpha_{i}=0(i=1,3)$ and $\beta_{n}=\lambda^{n} / n !(n=1,4)$. The 4thorder correction term is plotted in Fig. 7 as function of 


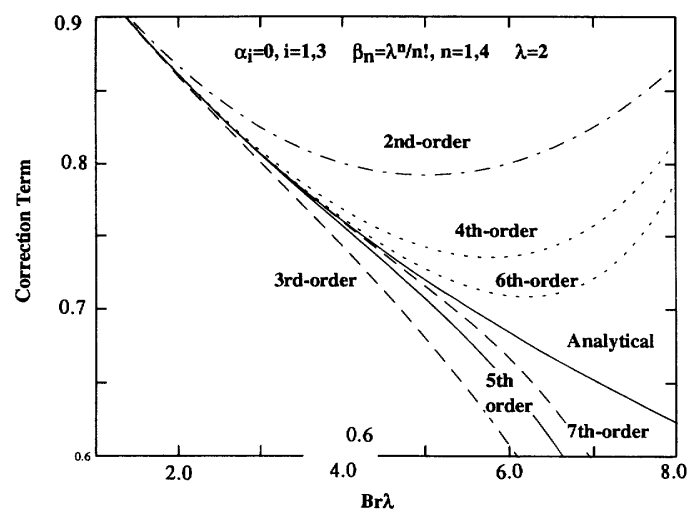

Fig. 8 Predictions of various series expansions of Eq. (46) for the wall shear rate as a function of $B r \lambda$

$B r \lambda$, for constant as well as temperature-dependent thermal conductivity. Also plotted are correction terms corresponding to lower-order solutions (obtained for constant thermal conductivity) as well as the correction term corresponding to Nahme's solution. Evidently, the third- and fourth-order solutions do offer an improvement, compared to the second-order result of Turian and Bird (1963). Inspection of Fig. 7 shows that the 4th-order solution is valid for $B r \lambda$ up to 3.5 , while the second-order solution is valid for $B r \lambda$ less than 2. It should be pointed out that, compared to the excellent agreement between the series solutions for velocity and temperature (Tables $1 \& 2$ and Fig. 4), the range of validity of the derived correction term is significantly truncated. This is to be anticipated, since the calculation of the wall shear rate involves differentiation of the velocity profile. This difficulty is not restricted to the series solution however: evaluation of wall shear rate from numerical solution of the governing equations is also subject to numerical differentiation errors that need to be carefully assessed. When the fluid thermal conductivity is allowed to change with temperature, the predicted magnitude of the correction term changes (curve 4.2 in Fig. 7). At $B r \lambda=3.5$ (where both solutions are anticipated to be reasonably correct), the constant $\kappa$ model prediction for the correction term is 0.787 while the prediction of the variable- $\kappa$-model is $0.796-\mathrm{a}$ discrepancy of $1.1 \%$.

It is instructive to examine the improvement in the accuracy of the calculation of the correction term that can be anticipated by solutions of higher-order in $\mathrm{Br}$. It has been shown previously that when the thermal conductivity is constant and the coefficients $\left(\beta_{i}\right)$ of the viscosity model are obtained as coefficients of a series expansion of the exponential (Eq. (35)), the series solution for the correction term obtained in this study is identical to a series expansion of the correction term resulting from Nahme's solution (Eq. (46)). Figure 8 compares the predictions of various- order expansions of Eq. (46) to Eq. (46) itself. Evidently, the rate of convergence to the "exact" result drops as the order of the expansion is increased. This seems to indicate that the extension in the range of validity caused by a higher-order expansion will, most probably, not be justified by the complexity of the resulting expressions.

\section{Conclusion}

Series solutions, up to $4^{\text {th }}$ order in the Brinkman number have been developed for planar Couette flow of materials whose viscosity and thermal conductivity can be expressed as polynomial functions of temperature with arbitrary coefficients. The derived solutions have been validated by extensive comparison to existing closed-form results and are found to be highly accurate for a practical range of the Nahme number (expressed by the product $B r \lambda$ ). The relative error in the predicted velocity profile is less than $0.5 \%$ for $B r \lambda<4.0$. The presented solutions provide valuable insight into the functionality of the relevant dependencies, something that is lost in purely numerical solutions. Based on these results, the distribution of velocity, shear rate and temperature, as well as their sensitivity on material and process parameters has been investigated. Formal corrections for viscosity measurements in Couette viscometers have also been derived. These account for the effect of viscous heating in the presence of temperature-dependent transport properties and have been found to be reliable for values of $B r \lambda$ up to 4.0.

\section{Appendix A:}

The coefficients $\Delta_{1}-\Delta_{9}$ appearing in the expressions for the velocity profile in planar Couette flow (Eq. (28)) are as follows:

$$
\begin{aligned}
\Delta_{9}= & \frac{1}{16} \cdot \beta_{4}+\frac{15}{448} \cdot \beta_{1} \cdot \beta_{3}-\frac{3}{32} \cdot \beta_{3} \cdot \alpha_{1}+\frac{253}{13440} \cdot \beta_{1}{ }^{2} \cdot \alpha_{1}{ }^{2} \\
& -\frac{5}{128} \cdot \beta_{1} \cdot \alpha_{1}{ }^{3}+\frac{1}{64} \cdot \beta_{1} \cdot \alpha_{3} \ldots \\
& +\frac{-9}{4480} \cdot \beta_{1}{ }^{3} \cdot \alpha_{1}-\frac{281}{6720} \cdot \beta_{1} \cdot \alpha_{1} \cdot \beta_{2}-\frac{5}{448} \cdot \beta_{1}{ }^{2} \cdot \alpha_{2} \\
& +\frac{9}{2240} \cdot \beta_{2} \cdot \beta_{1}{ }^{2} \ldots \\
& +\frac{5}{96} \cdot \beta_{1} \cdot \alpha_{2} \cdot \alpha_{1}+\frac{1}{40320} \cdot \beta_{1}{ }^{4}+\frac{5}{64} \cdot \alpha_{1}{ }^{2} \cdot \beta_{2} \\
& -\frac{1}{24} \cdot \alpha_{2} \cdot \beta_{2}+\frac{1}{120} \cdot \beta_{2}{ }^{2} \\
\Delta_{8}= & \frac{-1}{4} \cdot \beta_{4}-\frac{15}{112} \cdot \beta_{1} \cdot \beta_{3}+\frac{3}{8} \cdot \beta_{3} \cdot \alpha_{1}+\frac{281}{1680} \cdot \beta_{1} \cdot \alpha_{1} \cdot \beta_{2} \\
& +\frac{5}{32} \cdot \beta_{1} \cdot \alpha_{1}{ }^{3}-\frac{5}{24} \cdot \beta_{1} \cdot \alpha_{2} \cdot \alpha_{1} \ldots
\end{aligned}
$$




$$
\begin{aligned}
& +\frac{9}{1120} \cdot \beta_{1}{ }^{3} \cdot \alpha_{1}-\frac{253}{3360} \cdot \beta_{1}{ }^{2} \cdot \alpha_{1}{ }^{2}-\frac{1}{10080} \cdot \beta_{1}{ }^{4} \\
& -\frac{9}{560} \cdot \beta_{2} \cdot \beta_{1}^{2}+\frac{1}{16} \cdot \beta_{1} \cdot \alpha_{3} \ldots \\
& +\frac{5}{112} \cdot \beta_{1}^{2} \cdot \alpha_{2}-\frac{5}{16} \cdot \alpha_{1}^{2} \cdot \beta_{2}-\frac{1}{30} \cdot \beta_{2}^{2}+\frac{1}{6} \cdot \alpha_{2} \cdot \beta_{2} \\
& \Delta_{7}=\frac{-15}{64} \cdot \beta_{1} \cdot \alpha_{1}{ }^{3}+\frac{7}{8640} \cdot \beta_{1}{ }^{4}+\frac{7}{160} \cdot \beta_{2} \cdot \beta_{1}{ }^{2}-\frac{7}{320} \cdot \beta_{1}{ }^{3} \cdot \alpha_{1} \\
& -\frac{3}{32} \cdot \beta_{1} \cdot \alpha_{3}-\frac{29}{360} \cdot \beta_{1}^{2} \cdot \alpha_{2} \ldots \\
& +\frac{5}{16} \cdot \beta_{1} \cdot \alpha_{2} \cdot \alpha_{1}+\frac{29}{120} \cdot \beta_{1} \cdot \beta_{3}-\frac{23}{80} \cdot \beta_{1} \cdot \alpha_{1} \cdot \beta_{2} \\
& +\frac{127}{960} \cdot \beta_{1}^{2} \cdot \alpha_{1}^{2}+\frac{3}{8} \cdot \beta_{4}-\frac{9}{16} \cdot \beta_{3} \cdot \alpha_{1} \ldots \\
& +\frac{15}{32} \cdot \alpha_{1}^{2} \cdot \beta_{2}+\frac{11}{240} \cdot \beta_{2}{ }^{2}-\frac{1}{4} \cdot \alpha_{2} \cdot \beta_{2} \\
& \Delta_{6}=\frac{-1}{4} \cdot \beta_{4}-\frac{41}{160} \cdot \beta_{1} \cdot \beta_{3}+\frac{3}{8} \cdot \beta_{3} \cdot \alpha_{1}-\frac{2}{15} \cdot \beta_{1}{ }^{2} \cdot \alpha_{1}{ }^{2} \\
& -\frac{3}{40} \cdot \beta_{2} \cdot \beta_{1}^{2}+\frac{5}{32} \cdot \beta_{1} \cdot \alpha_{1}{ }^{3}+\frac{133}{480} \cdot \beta_{1} \cdot \alpha_{1} \cdot \beta_{2} \ldots \\
& +\frac{41}{480} \cdot \beta_{1}^{2} \cdot \alpha_{2}+\frac{1}{16} \cdot \beta_{1} \cdot \alpha_{3}-\frac{5}{24} \cdot \beta_{1} \cdot \alpha_{2} \cdot \alpha_{1} \\
& -\frac{1}{480} \cdot \beta_{1}{ }^{4}+\frac{3}{80} \cdot \beta_{1}{ }^{3} \cdot \alpha_{1} \ldots \\
& +\frac{1}{6} \cdot \alpha_{2} \cdot \beta_{2}-\frac{1}{48} \cdot \beta_{2}^{2}-\frac{5}{16} \cdot \alpha_{1}^{2} \cdot \beta_{2}
\end{aligned}
$$$$
\Delta_{5}=\frac{5}{32} \cdot \beta_{1} \cdot \beta_{3}-\frac{3}{32} \cdot \beta_{3} \cdot \alpha_{1}+\frac{1}{16} \cdot \beta_{4}+\frac{43}{576} \cdot \beta_{2} \cdot \beta_{1}{ }^{2}
$$$$
-\frac{43}{1152} \cdot \beta_{1}{ }^{3} \cdot \alpha_{1}+\frac{19}{3456} \cdot \beta_{1}{ }^{4} \ldots
$$$$
+\frac{29}{384} \cdot \beta_{1}^{2} \cdot \alpha_{1}^{2}+\frac{5}{96} \cdot \beta_{1} \cdot \alpha_{2} \cdot \alpha_{1}-\frac{7}{48} \cdot \beta_{1} \cdot \alpha_{1} \cdot \beta_{2}
$$$$
-\frac{5}{96} \cdot \beta_{1}^{2} \cdot \alpha_{2}-\frac{5}{128} \cdot \beta_{1} \cdot \alpha_{1}{ }^{3} \ldots
$$$$
+\frac{-1}{64} \cdot \beta_{1} \cdot \alpha_{3}-\frac{1}{96} \cdot \beta_{2}{ }^{2}+\frac{5}{64} \cdot \alpha_{1}^{2} \cdot \beta_{2}-\frac{1}{24} \cdot \alpha_{2} \cdot \beta_{2}
$$

$$
\begin{aligned}
\Delta_{4}= & \frac{-1}{24} \cdot \beta_{1} \cdot \beta_{3}-\frac{1}{60} \cdot \beta_{1}{ }^{2} \cdot \alpha_{1}{ }^{2}-\frac{11}{1440} \cdot \beta_{1}{ }^{4}-\frac{31}{720} \cdot \beta_{2} \cdot \beta_{1}{ }^{2} \\
& +\frac{1}{40} \cdot \beta_{1} \cdot \alpha_{1} \cdot \beta_{2}+\frac{1}{72} \cdot \beta_{1}{ }^{2} \cdot \alpha_{2} \ldots \\
& +\frac{31}{1440} \cdot \beta_{1}{ }^{3} \cdot \alpha_{1}+\frac{1}{60} \cdot \beta_{2}{ }^{2}
\end{aligned}
$$

\section{Appendix B:}

$$
\begin{aligned}
\Delta_{2}= & \frac{-1}{2240} \cdot \beta_{1}{ }^{2} \cdot \alpha_{1}{ }^{2}-\frac{37}{20160} \cdot \beta_{1}{ }^{3} \cdot \alpha_{1}+\frac{1}{1120} \cdot \beta_{1} \cdot \alpha_{1} \cdot \beta_{2} \\
& +\frac{1}{3360} \cdot \beta_{1}{ }^{2} \cdot \alpha_{2} \ldots \\
& +\frac{37}{10080} \cdot \beta_{2} \cdot \beta_{1}{ }^{2}-\frac{11}{3360} \cdot \beta_{1}{ }^{4}-\frac{1}{1120} \cdot \beta_{1} \cdot \beta_{3} \\
\Delta_{1}= & \frac{1}{2304} \cdot \beta_{1}{ }^{4}+\frac{11}{48384} \cdot \beta_{1}{ }^{2} \cdot \alpha_{1}{ }^{2}-\frac{1}{10080} \cdot \beta_{4} \\
& -\frac{13}{24192} \cdot \beta_{1} \cdot \alpha_{1} \cdot \beta_{2}-\frac{1}{12096} \cdot \beta_{1} \cdot \alpha_{2} \cdot \alpha_{1} \ldots \\
& +\frac{1}{2304} \cdot \beta_{1}{ }^{3} \cdot \alpha_{1}-\frac{1}{8064} \cdot \beta_{1}{ }^{2} \cdot \alpha_{2}+\frac{1}{6720} \cdot \beta_{3} \cdot \alpha_{1} \\
& -\frac{1}{1152} \cdot \beta_{2} \cdot \beta_{1}{ }^{2}+\frac{1}{16128} \cdot \beta_{1} \cdot \alpha_{1}{ }^{3} \ldots \\
& +\frac{1}{2688} \cdot \beta_{1} \cdot \beta_{3}+\frac{1}{6048} \cdot \beta_{2}{ }^{2}+\frac{1}{40320} \cdot \beta_{1} \cdot \alpha_{3} \\
& +\frac{1}{15120} \cdot \alpha_{2} \cdot \beta_{2}-\frac{1}{8064} \cdot \alpha_{1}{ }^{2} \cdot \beta_{2}
\end{aligned}
$$

The coefficients $\Gamma_{1}-\Gamma_{8}$ appearing in the expressions for the temperature profile in planar Couette flow (Eq. (32)) are as follows:

$$
\begin{aligned}
& \Gamma_{8}=\frac{1}{8} \cdot\left[\begin{array}{l}
\frac{253}{1680} \cdot \beta_{1} \cdot \alpha^{2}-\frac{5}{16} \cdot \alpha_{1}{ }^{3}-\frac{1}{8} \cdot \alpha_{3} \\
-\frac{9}{560} \cdot \beta_{1}{ }^{2} \cdot \alpha_{1}-\frac{43}{840} \cdot \beta_{2} \cdot \alpha_{1}-\frac{5}{56} \cdot \beta_{1} \cdot \alpha_{2} \ldots \\
+\frac{1}{140} \cdot \beta_{1} \cdot \beta_{2}+\frac{5}{12} \cdot \alpha_{2} \cdot \alpha_{1}+\frac{1}{5040} \cdot \beta_{1}{ }^{3}+\frac{1}{56} \cdot \beta_{3}
\end{array}\right] \\
& \Gamma_{7}=\frac{1}{7} \cdot\left[\begin{array}{l}
\frac{43}{240} \cdot \beta_{2} \cdot \alpha_{1}+\frac{35}{32} \cdot \alpha_{1}{ }^{3}-\frac{35}{24} \cdot \alpha_{2} \cdot \alpha_{1} \\
+\frac{9}{160} \cdot \beta_{1}{ }^{2} \cdot \alpha_{1}-\frac{253}{480} \cdot \beta_{1} \cdot \alpha_{1}{ }^{2}-\frac{1}{1440} \cdot \beta_{1}{ }^{3} \cdots \\
+\frac{-1}{40} \cdot \beta_{1} \cdot \beta_{2}-\frac{1}{16} \cdot \beta_{3}+\frac{7}{16} \cdot \alpha_{3}+\frac{5}{16} \cdot \beta_{1} \cdot \alpha_{2}
\end{array}\right]
\end{aligned}
$$




$$
\Gamma_{6}=\frac{1}{6} \cdot\left[\begin{array}{l}
\frac{-45}{32} \cdot \alpha_{1}{ }^{3}+\frac{1}{240} \cdot \beta_{1}{ }^{3}+\frac{11}{240} \cdot \beta_{1} \cdot \beta_{2}-\frac{19}{160} \cdot \beta_{1}{ }^{2} \cdot \alpha_{1} \\
-\frac{9}{16} \cdot \alpha_{3}-\frac{37}{80} \cdot \beta_{1} \cdot \alpha_{2}+\frac{15}{8} \cdot \alpha_{2} \cdot \alpha_{1} \ldots \\
+\frac{3}{40} \cdot \beta_{3}-\frac{17}{80} \cdot \beta_{2} \cdot \alpha_{1}+\frac{61}{80} \cdot \beta_{1} \cdot \alpha_{1}{ }^{2}
\end{array}\right]
$$

$$
\Gamma_{5}=\frac{1}{5} \cdot\left[\begin{array}{l}
\frac{-113}{192} \cdot \beta_{1} \cdot \alpha_{1}{ }^{2}-\frac{5}{96} \cdot \beta_{1} \cdot \beta_{2}-\frac{1}{32} \cdot \beta_{3}+\frac{25}{32} \cdot \alpha_{1}{ }^{3} \\
+\frac{1}{12} \cdot \beta_{2} \cdot \alpha_{1}+\frac{3}{8} \cdot \beta_{1} \cdot \alpha_{2} \ldots \\
+\frac{5}{16} \cdot \alpha_{3}-\frac{25}{24} \cdot \alpha_{2} \cdot \alpha_{1}-\frac{5}{576} \cdot \beta_{1}{ }^{3}+\frac{5}{32} \cdot \beta_{1}{ }^{2} \cdot \alpha_{1}
\end{array}\right]
$$

$$
\Gamma_{4}=\frac{1}{4} \cdot\left[\begin{array}{l}
\frac{1}{45} \cdot \beta_{1} \cdot \beta_{2}-\frac{9}{80} \cdot \beta_{1}{ }^{2} \cdot \alpha_{1}+\frac{11}{720} \cdot \beta_{1}{ }^{3} \\
+\frac{29}{120} \cdot \beta_{1} \cdot \alpha_{1}{ }^{2}+\frac{5}{24} \cdot \alpha_{2} \cdot \alpha_{1}+\frac{1}{60} \cdot \beta_{2} \cdot \alpha_{1} \ldots \\
+\frac{-1}{6} \cdot \beta_{1} \cdot \alpha_{2}-\frac{5}{32} \cdot \alpha_{1}{ }^{3}-\frac{1}{16} \cdot \alpha_{3}
\end{array}\right.
$$

$$
\Gamma_{3}=\frac{1}{3}\left(\begin{array}{l}
\frac{-3}{80} \cdot \beta_{1} \cdot \alpha_{1}{ }^{2}-\frac{7}{480} \cdot \beta_{1}{ }^{3}+\frac{1}{160} \cdot \beta_{1} \cdot \beta_{2} \\
-\frac{3}{160} \cdot \beta_{2} \cdot \alpha_{1}+\frac{1}{32} \cdot \beta_{1} \cdot \alpha_{2}+\frac{13}{320} \cdot \beta_{1}{ }^{2} \cdot \alpha_{1}
\end{array}\right)
$$

$$
\Gamma_{2}=\frac{1}{2} \cdot\left[\begin{array}{l}
\frac{-37}{5040} \cdot \beta_{1} \cdot \beta_{2}-\frac{1}{840} \cdot \beta_{1} \cdot \alpha_{1}{ }^{2}+\frac{1}{560} \cdot \beta_{3} \\
+\frac{1}{420} \cdot \beta_{2} \cdot \alpha_{1}-\frac{29}{6720} \cdot \beta_{1}{ }^{2} \cdot \alpha_{1} \ldots \\
+\frac{-1}{1680} \cdot \beta_{1} \cdot \alpha_{2}+\frac{11}{1680} \cdot \beta_{1}{ }^{3}
\end{array}\right.
$$

$$
\Gamma_{1}=\left(\begin{array}{l}
\frac{-1}{4480} \cdot \beta_{1} \cdot \alpha_{1}{ }^{2}-\frac{1}{1344} \cdot \beta_{1}{ }^{2} \cdot \alpha_{1}+\frac{1}{2240} \cdot \beta_{2} \cdot \alpha_{1} \\
+\frac{1}{6720} \cdot \beta_{1} \cdot \alpha_{2}+\frac{1}{672} \cdot \beta_{1} \cdot \beta_{2}-\frac{1}{896} \cdot \beta_{1}{ }^{3}-\frac{1}{2240} \cdot \beta_{3}
\end{array}\right)
$$

\footnotetext{
Nomenclature

$\mathrm{Br} \quad=$ brinkman number

$L \quad=$ gap of the Couette
}

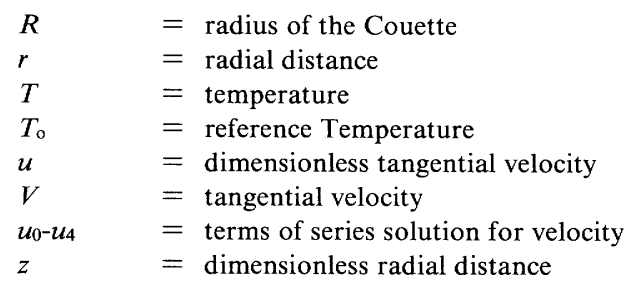

$\alpha_{i} \quad=$ coefficients in the thermal conductivity model (Eq. (4))

$\beta_{i} \quad=$ coefficients in the viscosity model (Eq. (5))

$\varepsilon \quad=$ eccentricity of the Couette (inner radius/outer radius)

$=$ thermal conductivity

$=$ coefficient in the exponential model for viscosity

$=$ viscosity

$\Theta \quad=$ dimensionless temperature

$\Theta_{1}-\Theta_{4}=$ terms of series solution for temperature

$\Omega \quad=$ angular velocity

\section{Literature Cited}

Agassant, J.F., P. Avenas, J.P. Segent and P.J. Carreau; Polymer Processing: Principles and Modelling, Hanser, Berlin (1991)

Bird, R.B., W.E. Stewart and E.N. Lightfoot; Transport Phenomena, John Wiley and Sons, NY, USA (1960)

Broer, L.J.F., personal communication to R.B. Bird (1958)

Campbell, G.S., J.D. Jungbauer, W.R. Bidlake and R.D. Hungerford; "Predicting the Effect of Temperature on Soil Thermal Conductivity," Soil Science, 158, 307-313 (1994)

Daivis, P.J and D.J. Evans; "Thermal Conductivity of a Shearing Molecular Fluid," Int. J. Thermophysics, 16, 391 (1995)

Gadala-Maria, F. and A. Acrivos; "Shear Induced Structure in Concentrated Suspensions of Solid Spheres," J. Rheol., 24, 799814 (1980)

Galvin, G.D., J.F. Hutton and B. Jones; “Development of HighPressure, High-Shear Rate Capillary Viscometer," J. NonNewtonian Fluid Mechanics, 8, 11-28 (1981)

Gavis, J. and R.L. Lawrence; "Viscous Heating in Plane and Circular Couette Flow Between Moving Surfaces," I\&EC Fundamentals, 7, 232-239 (1968)

Halliday, P.J., R. Parker, A.C. Smith and D.C. Steer; "The Thermal Conductivity of Maize Grits and Potato Granules," Journal of Food Engineering, 26, 273-288 (1995)

Harper, J.M.; Extrusion of Foods, CRC Press, Florida, USA (1981)

Holdsworth, S.D.; "Rheological Models used for the Prediction of the Flow Properties of Food Products: A Literature Review," Trans IChemE, 71, 139-179 (1993)

Jansen, K.M.B and J. van Dam, "An Analytical Solution for the Temperature Profiles During Injection Molding, Including Dissipation Effects," Rheol. Acta, 31, 592-602 (1992)

Joseph, D.D.; "Variable Viscosity Effects on the Flow and Stability of Flow in Channels and Pipes," Phys. Fluids, 7, 1761 (1964)

Joseph, D.D.; "Stability of Frictionally-Heated Flow," Phys Fluids, 8, 2195 (1965)

Ko, Y.S. and A.S. Lodge; "Viscous Heating Correction for Thermally Developing Flows in Slit-Die Viscometry," Rheol. Acta, 30, 357-368 (1991)

Laun, H.M.; "Polymer Melt Rheology with a Slit Die," Rheol. Acta, 22, 171-185 (1983)

Leighton, D. and A. Acrivos; "The Shear-Induced Migration of Particles in Concentrated Suspensions," J. Fluid Mechanics, 181, 415-439 (1987)

Nahme, R.; Ing.Arch., 11, 191 (1940)

Press, W.H., B.P Flannery, S.A. Teukolsky and W.T. Vetterling; Numerical Recipes: The Art of Scientific Computing, Cambridge University Press, England (1985)

Shadid, J.N. and E.R.G. Eckert; "Viscous Heating of a Cylinder 
with Finite Length by a High-Viscosity Fluid in Steady Longitudinal Flow: 2. Non-Newtonian Carreau Model Fluids," Int. J. Heat Mass Transfer, 35, 2739-2749 (1992)

Sukanek, P.C. and R.L. Lawrence; “An Experimental Investigation of Viscous Heating in Some Simple Shear Flows," AIChEJ., 20, 474-484 (1974)

Tsvetkov, O.B., Y.A. Laptev and A.G. Asambaev; "Thermal Conductivity of Refrigerants R123, R134a and R125 at Low Temperatures," Int. J. Thermophysics, 15, 203 (1994)
Turian, R.M. and R.B. Bird; "Viscous Heating in the Cone-andPlate Viscometer II," Chem. Eng. Sci., 18, 689-696 (1963)

Turian, R.M.; "Viscous Heating in the Cone-and-Plate Viscometer III," Chem. Eng. Sci., 20, 771-781 (1965)

Turian, R.M.; "The Critical Stress in Frictionally Heated NonNewtonian Plane Couette Flow," Chem. Eng. Sci., 24, 1581-1587 (1969) 